\title{
Enhancing Data Centre Networking Using Energy Aware Optical Interconnects
}

\author{
Ivan Glesk, Senior Member, IEEE Tolulope Osadola Member, IEEE, Siti Idris \\ Department of Electronic and Electrical Engineering, University of Strathclyde, Glasgow UK. \\ Tel: (+44) 141-548-2529, Fax: (+44) 141-552-4968, e-mail: ivan.glesk@strath.ac.uk
}

\begin{abstract}
In a fast changing world where information technology drives economic prosperity, the number of data centres has grown significantly in the past few years. These data centres require large amount of energy in order to meet up with increasing demands. An overview of energy efficient optical interconnects as a means of reducing energy consumption without compromising speed and accuracy was presented. New methods by which energy efficiency can be achieved using OCDMA multiplexing techniques for future optical interconnections were discussed. We also presented some challenges that might inhibit effective implementation of the OCDMA multiplexing scheme.
\end{abstract}

Keywords: Energy Efficiency, Optical Interconnects, Datacentre networking, Optical Switching, OCDMA

\section{INTRODUCTION}

As a result of recent developments in computing technology, more data enters are being built to accommodate both current and future demand for high speed computing [1]. Specifically, cloud computing has resulted in the need for large data centres to facilitate a consolidation of compute resources for processing and storage in both internet based (public) and enterprise (private) networks. The applications that are hosted in these data centers are contained in several servers and these applications could be cloud based applications, search engines, specialized software, etc.). They are extremely data-intensive and require constant communication between the numerous servers (storage servers, database servers, etc) in these data centers. This data transfers creates the need for high bandwidth and low latency communication networks between the servers in the data centers. As the numbers of these datacenters grow, service providers will continue to face the challenge of having to meet up with customer demand thereby creating a need for compute devices with higher processing power that can get the job done at the shortest possible time in order to maximize profit [2]. However, another challenge will be how to maximize the available energy resources in a way that will ensure sustainability. A device in a network can be regarded to be energy efficient if its energy consumption is proportional the amount of data it processes. In a datacenter, networking devices require about $15 \%$ of the total energy consumption [3]. Therefore, it is important that the amount of data transferred through the network infrastructure is large enough to justify this energy requirement. Electronic interconnect systems are limited by their communications infrastructure and the associated power dissipation at high-bandwidth. With the recent developments in nanoscale silicon photonics there is increasing potential for the development of energy efficient optical interconnection networks that will ultimately improve performance-per-Watt of compute devices. Nano-scale integrated Silicon photonic devices, offer many relevant advantages to the construction of these networks, including high bandwidth achieved using wavelength division multiplexing, optical time division multiplexing (OTDM) or optical code division multiplexing (OCDMA). Recently, a novel hybrid Silicon-Plasmonics components/subsystems for the demonstration of a $\mathrm{Tb} / \mathrm{s}$ scale router with ultralow power consumption, footprint and end-to-end propagation delay was reported [4]. In this paper we discuss recent developments in optical interconnection technologies towards achieving high speed-energy efficient networking. We analyze some potential multiplexing techniques and architectures that can potentially be implemented in future optical interconnect devices in order to achieve efficient traffic aggregation and ensure energy sustainability. We will also discuss the effects of environmental factors that might potentially mitigate the effective transmission of optical data.

\section{ENERGY EFFICIENT DATA CENTRE NETWORKS}

A data centre network consist of series of layers which are either physically or logically linked together to facilitate the transfer of information from one point to another. The servers which are usually arranged into racks are connected through a Top-of-the-Rack Switch (ToR). Traffic from these ToR switches are then aggregated by connecting them in a tree topology using links of higher capacity than the individual server links using aggregate switches. In larger networks, other layers can be used to aggregate the links further in order to ensure effective data transfer and also ensure reliability [5]. All of the devices /nodes in the network require switch ports with which they connect to the network. These ports serve as information conduits therefore the rate at which the ports are able to make decisions on information bits arriving at the ports, will determine the speed of execution of information request and compute demands. One disadvantage of current implementations of the layered architectural model of the data centre is that most of these high capacity electronic switches consume enormous 
amount of energy because of the many ports that they use to aggregate data from several servers. Also, in order to make forwarding decisions on data, it has to be first converted from the high speed optical domain to electrical domain and vice versa, this conversion process can create a bottleneck situation in electronic switches because of the limit in the speed of electronic processing. Several methods of eliminating the electronic bottleneck have been proposed [6] with the ultimate aim of achieving a transparent network architecture that uses high speed low energy photonic switches. In [7], a modular optical packet switching (OPS) architecture for network interconnects in the data centre by employing highly distributed control was presented experimentally. The 8 input-8 output optical packet switch module with capable of operating at $40 \mathrm{~Gb} / \mathrm{s}$ data rate was found to consume a record low energy of less than $76.5 \mathrm{pJ} / \mathrm{bit}$. Example of commercially available all optical switch is the Polatis optical interconnect solution for data centre which comprise of a reconfigurable optical switch based on piezoelectric switching technology. It also incorporates beam steering technology. Some methods have been proposed in order to optimize the power consumption of data centres network devices these include powering off unused links and switches while maintaining performance and fault tolerance goals, however, the Polatis solution has been reported to have a power consumption of about $1 / 20^{\text {th }}$ of the power consumed by legacy electronic switches of comparable capacity [8]. Because these photonic switches are capable of making decisions all optically, there will be no need for energy consuming opto-electro-opto conversion ports and also, because of the higher speed of photonic switches relative to electronic switches, bottlenecks can be completely eliminated. Even as these devices becomes faster and latency is reduced, there still exists concerns about the energy efficiency of these interconnect devices. The energy efficiency of a novel data centre network architecture based on optical multiple-input multiple-output (MIMO) orthogonal frequency division multiplexing (OFDM) technology was evaluated in [9]. The architecture was reported to offer higher energy efficiency when compared with a reference design based on commodity electrical switches.

\section{NOVEL POWER SAVING ARCHITECTURE FOR OCDMA CODE GENERATION}

Optical code division multiple access (OCDMA) is one possible family of optical multiple access techniques for the next generation broadband networking which has attracted considerable attention in the past decade. Because of its potential for high speed asynchronous all - optical transmission of data it can be adapted for use in a datacentre networking. Fiber Bragg grating (FBG) optical reflectors have become quite popular for the purpose of encoding and decoding optical code division multiple access (OCDMA) data. When the source of optical signal is a broadband supercontinuum source, the source signal is traditionally distributed equally to all users as shown in architecture in fig. 1.

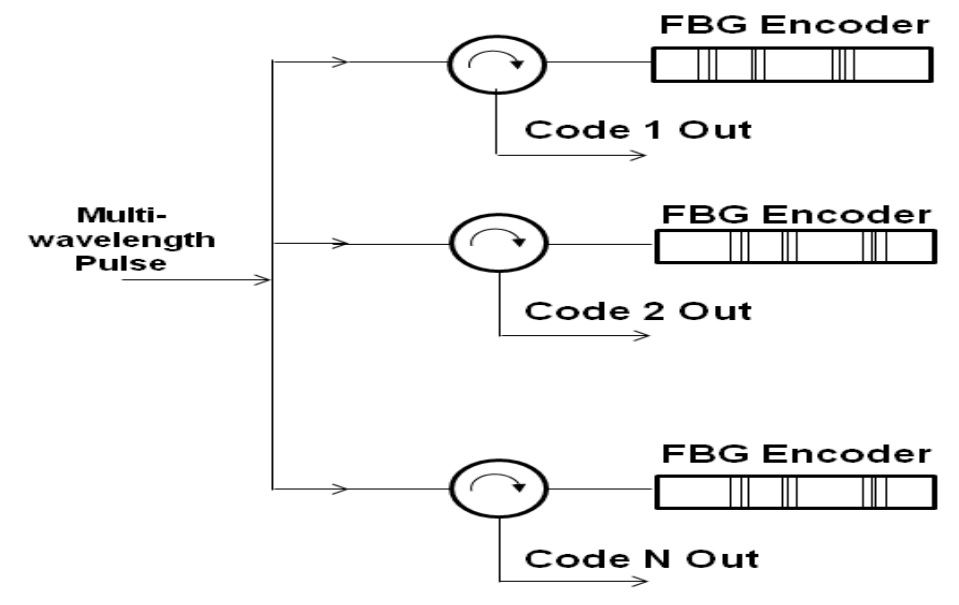

Fig. 1. Traditional method of generating OCDMA signals from a multiwavelength pulse

However, we observed that only about $20 \%$ of the power that enters into an optical en/decoder is reflected and the remaining $80 \%$ is not used in the en/decoding process. We present a novel architecture that will enable both cost and energy efficiency in the process of generating optical CDMA codes. The architecture achieves effective utilization of available optical power by using several cascaded OCDMA encoders. In this new architecture (see Fig. 2), each OCDMA encoder is supplied with multiwavelength optical source by the encoder preceding it. 


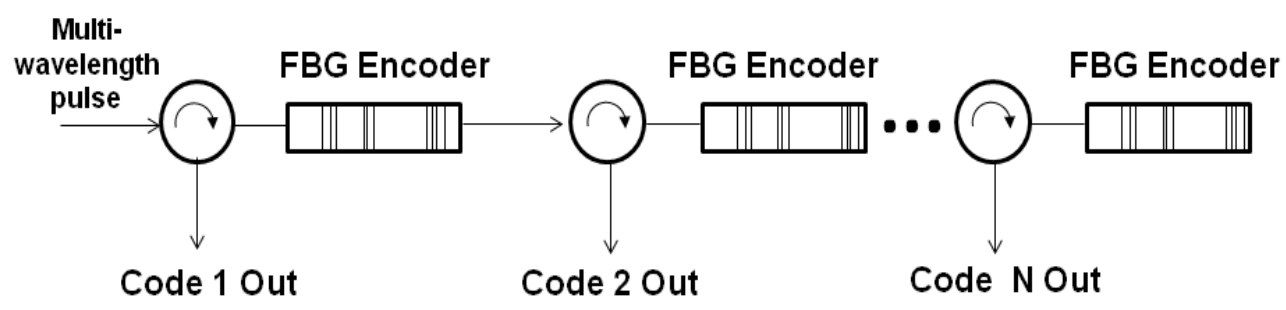

Fig. 2. Proposed cascade method that enables effective utilization of optical power

Using the architecture in fig. 2 instead of the traditional method as depicted in fig.1, we have been able to achieve power savings of up to $3 \mathrm{~dB}$ for a 4- user OCDMA system. We were also able to eliminate the use of the initial power splitter.

\section{CHALLENGES OF OCDMA FOR INTERCONNECT APPLICATIONS}

Because of the nature of the datacentre environment, it is necessary to consider some factors when implementing OCDMA in a datacentre network. Recently, we studied of the effect of environmental temperature variation on the bit error rate (BER) performance of multiwavelength 2-dimensional wavelength hopping time spreading optical code division multiple access (2D-WH/TS OCDMA) signals that utilises picosecond pulses to form incoherent optical codes. Using equations already derived in [10] for modelling the effects of temperature variation on autocorrelation signal resulting from the decoding of an incoherent 2D-WH/TS OCDMA encoded signal which consists of $w$ wavelength pulses each having a pulsewidth of $\tau$ after propagating in $\mathrm{L}(\mathrm{Km})$ of fibre, we arrive at the expression for the envelope of the resulting autocorrelation peak St.

$$
S_{t}(L)=\sum_{k=0}^{w-1} P_{p} \exp \left\{-2.77\left[\frac{t-k \Delta t}{\tau-\Delta \tau}\right]^{2}\right\}
$$

Where

$$
\Delta t=D_{\text {temp }} \times \Delta T \times \Delta \Lambda \times L \text { and } \Delta \tau=D_{\text {temp }} \times \Delta T \times \Delta \lambda \times L
$$

Where $D_{\text {temp }}(\mathrm{ps} / \mathrm{nm} \bullet \mathrm{km} / \mathrm{oC})$ is the thermal coefficient of the fiber $[11,12], \Delta T\left({ }^{\circ} \mathrm{C}\right)$ is the average change in temperature experienced by optical fiber, $\Delta \Lambda$ is the spectral spacing between 2D-WH/TS OCDMA code wavelengths pulses, and $\Delta \lambda(\mathrm{nm})$ is the pulse spectral line width of each wavelength pulse within the code. Having obtained the maximum possible autocorrelation peak St for each degree of temperature change, we analysed the effect of this reduction in St with respect to temperature variation by substituting St for th in the equation for Pe (BER) as previously derived in [1] and we obtain the equation below

$$
P_{e}=\frac{1}{2} \sum_{j=0}^{S t}(-1)^{\mathrm{j}}\left(\begin{array}{c}
w \\
j
\end{array}\right)\left(1-\frac{j q_{1,1}}{w}\right)^{K-1}
$$

Where $q_{1,1}$ is the hit probability and is given as

$$
q_{1,1}=\frac{\text { Code weigth }(w)}{2 x \text { Code length }(N c)}
$$

Figure 3 shows the envelope of St for an 8 wavelength 2D-WH/TS OCDMA signal after propagation in a 10km optical fibre link ( $\mathrm{ps} / \mathrm{nm} \cdot \mathrm{km} /{ }^{\circ} \mathrm{C}$, and , $\mathrm{Nc}=$ code length $)$ with initial pulsewidth of $2 \mathrm{ps}$. Three different scenarios have been illustrated in the figure for $\Delta \mathrm{T}=0,10$ and 20 degrees respectively. To evaluate the effect of the temperature variation induced reduction in $\mathrm{St}$, the minimum possible bit error rate performance for $\mathrm{K}=32$ simultaneous users at $2.4 \mathrm{~Gb} / \mathrm{s}$ data rate was recorded from calculations obtained using Eq. 2 for $\Delta \mathrm{T}$ between 0 and $20^{\circ} \mathrm{C}$ over a $10 \mathrm{~km}$ and $20 \mathrm{~km}$ fiber optic link. The results are presented in Figure 4 . We found that trade-offs must be made between number of simultaneous users and transmission distance in order to maintain performance. 


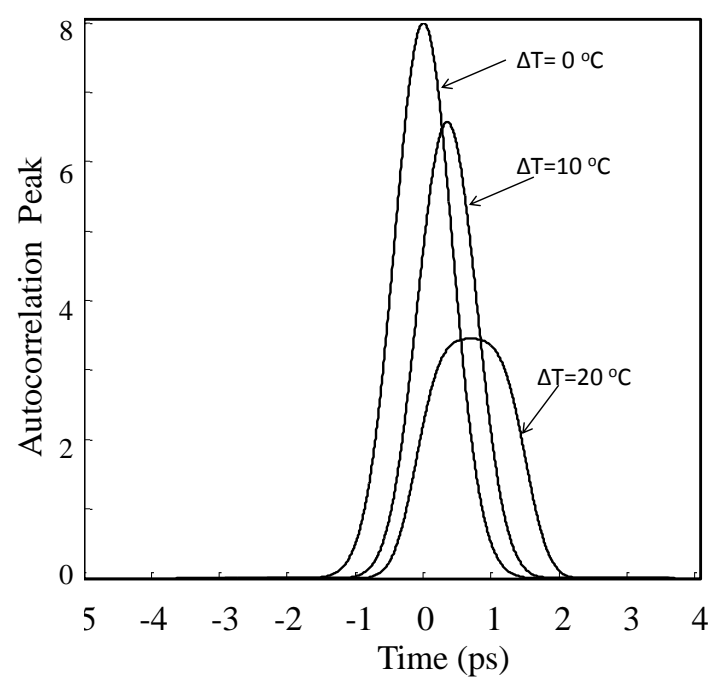

Fig.3 Maximum obtainable autocorrelation peak for different values of $\Delta \mathrm{T}$ at a link span of $10 \mathrm{~km}$

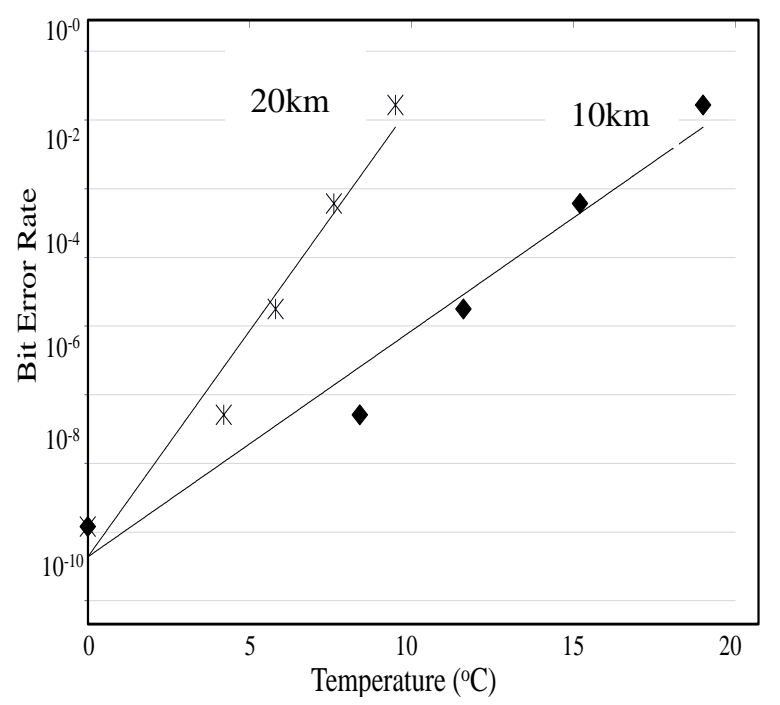

Fig.4 Minimum obtainable Bit Error Rate as $\Delta \mathrm{T}$ increases over a $10 \mathrm{~km}$ link.

\section{CONCLUSIONS}

We have discussed the role of optical interconnects in improving the overall energy efficiency of datacentres. We highlighted several implementations of optical switching and observed that optical packet switches will not only eliminate electronic bottlenecks in data transfer but can also perform switching at faster speeds with record low energy usage compared to electronic methods. Finally we presented some of our findings on the potential use of OCDMA for energy efficient data centre networking.

\section{REFERENCES}

[1] A. Kasukawa, K.Takaki, S. Imai, H. Shimizu, Y. Kawakita,; K. Hiraiwa, M. Funabashi, T. Suzuki, N.Tsukiji, S. Kamiya, T. Ishikawa, "Enabling VCSEL technology for "Green" optical interconnect in HPC and Data Centers," Photonics Conference (PHO), 2011 IEEE, vol., no., pp.393,394, 9-13 Oct. 2011

[2] H. Wang, A. S. Garg, K. Bergman, and M. Glick, "Design and demonstration of an all-optical hybrid packet and circuit switched network platform for next generation data centers," in Optical Fiber Communication Conference. Optical Society of America, 2010, p.OTuP3.

[3] A. Schlosser, L. Stobbe, N.F. Nissen, B. Schappi, K. Lang, "Energy efficient network equipment for data centers," Electronics Goes Green 2012+ (EGG), 2012 , vol., no., pp.1,5, 9-12 Sept. 2012

[4] K. Vyrsokinos, S. Papaioannou, D. Kalavrouziotis, J. Weeber, K. Hassan, L. Markey, A. Dereux, A. Kumar, S.I. Bozhevolnyi, M. Baus, G. Giannoulis, D. Apostolopoulos, H. Avramopoulos, N. Pleros, "Low energy routing platforms for optical interconnects using active Plasmonics integrated with Silicon Photonics," Transparent Optical Networks (ICTON), 2012 14th International Conference on , vol., no., pp.1,4, 2-5 July 2012

[5] Kachris, Christoforos; Tomkos, Ioannis, "A Survey on Optical Interconnects for Data Centers," Communications Surveys \& Tutorials, IEEE, vol.14, no.4, pp.1021,1036, Fourth Quarter 2012

[6] H. Wang, A. S. Garg, K. Bergman, and M. Glick, "Design and demonstration of an all-optical hybrid packet and circuit switched network platform for next generation data centers," in Optical Fiber Communication Conference. Optical Society of America, 2010, p.OTuP3.

[7] N. Calabretta, L. Jun S.D. Lucente, H. Dorren, "Experimental assessment of low latency and large port count OPS for data center network interconnect," Transparent Optical Networks (ICTON), 2012 14th International Conference on , vol., no., pp.1,4, 2-5 July 2012

[8] The New Optical Data Center, Polatis Data Sheet, Polatis Inc., 2009

[9] P.N. Ji, C. Kachris, I. Tomkos, T. Wang, "Energy efficient data center network based on a flexible bandwidth MIMO OFDM optical interconnect," Cloud Computing Technology and Science (CloudCom), 2012 IEEE 4th International Conference on , vol., no., pp.699,704, 3-6 Dec. 2012.

[10] T. Osadola, S. Idris, I. Glesk, and W. Kwong, "Effect of Variations in Environmental Temperature on 2DWH/TS OCDMA Code Performance," J. Opt. Commun. Netw. 5, 68-73 (2013).

[11] G. Ghosh, M. Endo, and T. Iwasaki, "Temperature-dependent Sellmeier coefficients and chromatic dispersions for some optical fiber glasses," IEEE J. Light. Technol., 12, pp.1338-1342 (1994). 